\title{
Sustainability and its relation to efficiency under uncertainty
}

\author{
Frank C. Krysiak
}

Received: 27 April 2007 / Accepted: 26 June 2008 / Published online: 19 July 2008

(C) Springer-Verlag 2008

\begin{abstract}
Evaluating the long-run consequences of present actions, as in the context of sustainability, requires information about the actions' outcomes and about future preferences that is often uncertain. We analyze a risk-based criterion of sustainability and a corresponding efficiency concept that cover these uncertainties. We derive several properties of these criteria and formally characterize the trade-off between sustainability and efficiency. Furthermore, we show that maximizing the probability of ex post efficiency under a sustainability constraint provides an interesting choice rule and that, for a special case, this rule is connected to portfolio theory.
\end{abstract}

Keywords Sustainability · Efficiency · Uncertainty · Policy evaluation · Portfolio choice

\section{JEL Classification Q01 - D81 - D63 · G11}

\section{Introduction}

One important aspect of economics is to provide information about the relative merits of competing actions or projects. These are usually judged according to their effects on the welfare of the affected individuals. Traditionally, the main focus has been on evaluating and comparing actions that affect presently living individuals. But during the past decade, the effects of present actions on future individuals have increasingly been considered. A particular concept for this is sustainability, which holds that present

We are indebted to an anonymous referee whose comments helped substantially to improve the article.

F. C. Krysiak ( $\varangle)$

Department of Economics, University of Basel, Petersgraben 51, 4003 Basel, Switzerland

e-mail: Frank.Krysiak@unibas.ch 
actions should "not compromise the ability of future generations to meet their needs" (Brundtland 1987).

Such an intergenerational choice problem has several characteristics that set it apart from the problems traditionally analyzed in welfare economics and social choice theory. Most importantly, future individuals are not yet in existence. So their preferences are unknown at the time when decisions that influence their welfare have to be made. Also, it is frequently impossible to predict the future outcomes of present actions with certainty. Therefore, a long-run intergenerational setup demands that we evaluate projects under outcome and preference uncertainty.

But most of the literature devoted to evaluating the long-run consequences of present actions does not consider these uncertainties. Existing studies frequently employ tools that have been developed for the traditional intratemporal setup. Thereby, some interesting aspects are lost.

Most studies assume that future preferences are known with certainty today and, for the most part, that they are identical to present preferences. Therefore, these studies neglect the conceptually interesting aspect that sustainability implies planning for people that we do not know. Furthermore, neglecting preference uncertainty has facilitated the use of welfare comparisons between present and future individuals. As Krysiak and Krysiak (2006) argued, this becomes impossible if there is substantial uncertainty w.r.t. future preferences. So an evaluation of the long-term consequences of a project should avoid intergenerational welfare comparisons.

Also, neglecting uncertainty has led to the perhaps unwarranted assumption that we are able to assure that we do not harm future individuals. Under uncertainty, assuring such a deterministic version of sustainability is at least costly (in terms of foregone present and expected future welfare) if not even impossible. So the focus should rather be on balancing the risk of harming future individuals with the risk of rejecting projects that are beneficial to present and potentially to future individuals. This indicates that under uncertainty, the relation between sustainability and efficiency is important and should be addressed in terms of risks.

In this article, we consider the question of how the consequences of present actions for future individuals can be evaluated if future preferences and outcomes are uncertain and if intergenerational welfare comparisons are infeasible. For this, we use the riskbased concept of sustainability advanced in Krysiak and Krysiak (2006) as well as a similar risk-based efficiency criterion. We first prove some general characteristics of these concepts. We then investigate the relation between sustainability and efficiency and analyze a choice rule that minimizes the conflict between these criteria.

Our results indicate that such a risk-based approach provides a content-rich and yet mathematically convenient framework for analyzing decision problems with longrun consequences. The main definitions on which our analysis is based are shown to have mathematically convenient convexity and continuity properties that facilitate their integration into economic analysis. Furthermore, although there is an inherent conflict between sustainability and efficiency, our risk-based framework allows to replace the incompatibility of these concepts often found in deterministic models with a gradual choice between an efficiency-focused and a distribution-centered approach to intertemporal planning. Finally, minimizing the risk of ex post inefficiency under a sustainability constraint provides an economically intuitive and mathematically 
convenient choice rule with a connection to the efficient frontier of mean-variance analysis.

In the following two sections, we briefly review the literature on sustainability under uncertainty, advance our framework, and analyze a risk-based concept of sustainability. In Sect. 4, we examine the trade-off between sustainability and efficiency. In Sect. 5, we consider a special case in which our analysis can be connected to portfolio theory. Section 6 discusses the limitations of our analysis as well as some generalizations, Sect. 7 provides an example, and Sect. 8 concludes the article.

\section{Sustainability under uncertainty}

Several groups of studies have considered uncertainty in the context of sustainability or, more general, in the context of evaluating policies with long-run consequences.

Going back to Arrow and Fisher (1974) and Fisher and Krutilla (1974), there is an extensive literature that analyzes the effects of uncertainty in the context of project evaluation. This literature shows that the conjunction of uncertainty and irreversibility implies the existence of a quasi-option value, which can have a substantial influence on a project's preferability. However, these studies proceed in an aggregative framework that abstracts from distributional concerns. Thus, they are only distantly connected to the intergenerational distribution problem analyzed here.

Closer to our framework are studies that analyze sustainability under outcome uncertainty, such as Woodward (2000) and Asheim and Brekke (2002), or preference uncertainty, such as Heal et al. (1998), Ayong Le Kama (2001), Ayong Le Kama and Schubert (2004), and Krysiak and Krysiak (2006). Asheim and Brekke (2002) considered outcome uncertainty by introducing stochastic changes to capital and resource stocks into a concept of non-declining welfare. ${ }^{1}$ Woodward (2000) also addresses outcome uncertainty but uses a concept of sustainability that is based on the notion of fairness advanced in Foley (1967). ${ }^{2}$ Heal et al. (1998), Ayong Le Kama (2001), and Ayong Le Kama and Schubert (2004) derived decision rules for sustainable development for a special case of preference uncertainty where preferences change in an uncertain direction at a (possibly) uncertain future time. Finally, Krysiak and Krysiak (2006) analyzed an envy-based criterion of sustainability in the context of a more general form of preference uncertainty.

Albeit these studies introduce uncertainty either as outcome or as preference uncertainty, this difference is in itself not substantial from a conceptual point of view; whether future well-being is uncertain due to unpredictable future consumption possibilities or due to unpredictable future preferences is merely a question of model details.

However, Krysiak and Krysiak (2006) argued that this holds only for limited forms of preference uncertainty. If future preferences can deviate substantially from present

\footnotetext{
${ }^{1}$ Non-declining welfare is axiomatically derived as a criterion of sustainability in a deterministic context in Asheim et al. (2001). The underlying approach to intergenerational justice is extended in Asheim and Tungodden (2004).

2 Similar criteria are also used in Riley (1980) and Howarth (1995).
} 
preferences, it is not adequate to presume that future and present generations share a concept of well-being. Thus, welfare comparisons across generations are not feasible if there is substantial preference uncertainty. To avoid such comparisons, they advance a definition of sustainability that requires that the probability of harming future individuals is limited. Thereby, harm is defined as envy w.r.t. a status-quo situation, so that this definition does not rely on intergenerational welfare comparisons.

The concept is similar to that used in Woodward (2000) but uses a one-way notion of fairness (it is not required that present individuals do not envy future individuals), is based on actual outcomes and preferences instead of expected future welfare (and therefore corresponds to a different ethical view on intergenerational risk sharing), and avoids intergenerational welfare comparisons. Krysiak and Krysiak showed that this criterion of sustainability generalizes weak and strong sustainability as well as non-declining welfare. They argued that this approach is preferable to an expectedutility-based concept because it encompasses frequently used sustainability concepts and because the risk of harming future individuals can approach 100\% in the expected utility framework, which seems to be ethically untenable.

In this article, we use the sustainability concept advanced in Krysiak and Krysiak (2006) for three reasons. First and most importantly, it avoids intergenerational welfare comparisons and is thus applicable in the context of substantial preference uncertainty. Second, it encompasses several frequently used sustainability concepts. Finally, as we show below, it provides an interesting possibility to characterize the trade-off between sustainability and efficiency.

\section{Characterizing sustainable actions}

We consider a setting where a present decision influences the welfare of future individuals but where this influence cannot be predicted with certainty because both the effects of the present decision on future situations and the preferences according to which future individuals will assess their situation are uncertain. For simplicity, we restrict our analysis to the case where there is only one future generation that consists of a single individual and where there is only one presently living individual. As we argue in Sect. 6, extending our analysis to more applicable settings is easily possible.

Let $\tilde{\mathcal{A}} \subset \mathbb{R}^{n}$ be a bounded set of a finite number of actions. We assume that in addition to these actions, all their convex combinations are feasible. If we analyze, for example, a land-use decision, this assumption implies that the land can be partitioned and each partition used for a different alternative. So the set of feasible actions is the convex hull of $\tilde{\mathcal{A}}$, which we denote by $\mathcal{A}$. Observe that by construction, $\mathcal{A}$ is convex and compact.

Let denote by $s \in \mathcal{S} \subseteq \mathbb{R}^{q}$ a random variable that describes the future state of nature. Let $t \in \mathcal{T} \subseteq \mathbb{R}^{p}$ be the type of the future individual, which is also uncertain from the perspective of the present. We depict the preferences of the future individual over the outcomes of present actions in dependency of the state of nature and the type of the individual by defining a preference relation $\succ$ on $\mathcal{A} \times \mathcal{S} \times \mathcal{T}$, which is similar to the master preferences used in Howe (1987). Furthermore, we assume that a function $P(\mathcal{Q})$ exists that measures the probability that $(s, t) \in \mathcal{Q} \subseteq \mathcal{S} \times \mathcal{T}$ and 
that this probability has a density $\phi(s, t)$, which implies that $P$ is differentiable and thus continuous. For notational simplicity, we define $z:=(s, t)$ and $\mathcal{Z}:=\mathcal{S} \times \mathcal{T}$.

This notational simplification might suggest that our model is equivalent to a model with only outcome uncertainty. However, there is an important difference. If only outcomes are uncertain, welfare comparisons over differing values of $z$ involve only a comparison of different states. With preference uncertainty, they can involve intergenerational welfare comparisons, which are infeasible if there is substantial preference uncertainty. Thus although the distinction between outcome and preference uncertainty is notationally dispensable, it is important to keep in mind that, in contrast to a model with only outcome uncertainty, we cannot compare welfare levels across all elements of $z$ because we have to avoid intergenerational welfare comparisons.

As discussed in the preceding section, a possible criterion for sustainability that avoids intergenerational welfare comparisons and that is applicable to the context of uncertainty is given in Krysiak and Krysiak (2006). Following this approach, we define sustainability as the obligation to keep the probability of harming future individuals within reasonable bounds. Thereby, a future individual is "harmed" whenever an action results in an outcome that is, according to the preferences of this individual, strictly inferior to a status-quo.

We do not elaborate on the choice of this status-quo, because for our formal analysis, it suffices that an action that result in this status quo can be defined. Note, however, that together with setting the level of sustainability, which we introduce below, the choice of the status-quo action determines the ethical implications of this concept of sustainability. Since in many cases, the status-quo action will correspond to an action that leaves the distribution of the future states of the world unchanged, we denote it by 0 .

So, a future individual of type $t$ is "harmed" by the action $a$ in state $s$, if $(0, z) \succ$ $(a, z)$ with $z=(s, t)$, that is, if the individual would strictly prefer the status-quo outcome to the outcome of the action given the future state of nature $s$. The probability that the action $a$ harms the future individual is given by

$$
\omega(a):=P(\Sigma(a))
$$

with

$$
\Sigma(a):=\{z \in \mathcal{Z} \mid(0, z) \succ(a, z)\}
$$

Following Krysiak and Krysiak (2006), we define sustainability as the requirement that the probability of harming the future individual is bounded by a constant $\alpha$. Thus, for a given value of $\alpha$, the set of sustainable actions is

$$
\Omega_{\alpha}:=\{a \in \mathcal{A} \mid \omega(a) \leq \alpha\}
$$

To characterize $\Omega_{\alpha}$, we use the following assumptions:

A1 Preferences are complete, transitive, and continuous w.r.t. $(a, z) \in \mathcal{A} \times \mathcal{Z}$.

A2 If $\left(a_{1}, z_{1}\right) \succeq\left(b, z_{1}\right)$ and $\left(a_{2}, z_{2}\right) \succeq\left(b, z_{2}\right)$, then $\left(\lambda a_{1}+(1-\lambda) a_{2}, \lambda z_{1}+\right.$ $\left.(1-\lambda) z_{2}\right) \succeq\left(b, \lambda z_{1}+(1-\lambda) z_{2}\right)$ for all $\lambda \in[0,1]$. 
A3 $\mathcal{Z}$ is a convex set that contains at least two elements.

A4 The density $\phi(z)$ is convex unimodal, that is, for all $c \geq 0$, the level sets $\{z \in$ $\mathcal{Z} \mid \phi(z) \geq c\}$ are either empty or convex sets.

For some results, we need stronger conditions.

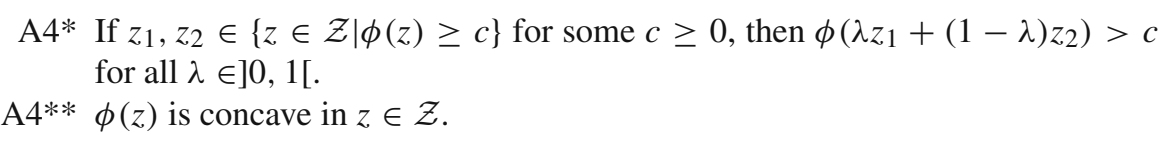

Assumption A1 is standard in economic analysis. A2 restricts the influence of outcome and preference uncertainty on the future evaluation of the outcomes of present actions. It implies that if there are two actions $a_{1}, a_{2}$ that are weakly preferred to an action $b$ for some future state and type combinations $z_{1}$ and $z_{2}$, respectively, then a convex combination of these actions is preferred to $b$ for the corresponding convex combination of the states and types. This assumption holds, for example, if preferences are convex w.r.t. $a$ and quasi-linear w.r.t. $z$.

Assumption A3 implies that if two states or two types are possible, a convex combination of these states or types is not impossible per se.

Assumption A4 is one of several extensions of the concept of a unimodal density to the multivariate case, see Dharmadhikari and Joag-Dev (1988). It excludes cases in which several distinct combinations of future states and types are likely with "in between" scenarios being less probable. It implies that $\phi(z)$ is a quasi-concave function of $z \in \mathcal{Z}$.

The stronger assumption A4* guarantees that the density of the probability distribution has a unique maximum, that is, there is a single scenario that is regarded as the most likely one. It rules out "plateaus" of $\phi(z)$ by demanding that the interior of any level set is a subset of some higher level set. But A4* does not specify how strongly the likelihood of a scenario decreases, if we move away from the most plausible scenario. Such a constraint is implicit in A4**, which sets a lower bound to this decrease; the likelihood of a scenario can at most change linearly with the distance to any other scenario. An example for a distribution that meets $4^{*}$ is the normal distribution. Examples for $\mathrm{A} 4 * *$ are the uniform and the triangular distribution, the latter of which meets both $\mathrm{A} 4 *$ and $\mathrm{A} 4 * *$. Note that both $\mathrm{A} 4 *$ and A4** imply A4, but neither of them implies or excludes the other. Furthermore, A4* does not constrain $\mathcal{Z}$, whereas A $4 * *$ is only feasible if this set is bounded.

Given the above assumptions, it is clear that the essential aspect of our setup is the convexity of all relevant sets, functions, and relations. Assumption A3 and our construction of $\mathcal{A}$ ensure that the set of action, the set of types, and the set of states of natures are convex. Assumption A2 is most easily met if preferences are convex in $a$ and quasi-linear in $z$. Assumptions $\mathrm{A} 4, \mathrm{~A} 4 *$, and $\mathrm{A} 4 * *$ imply that the density function $\phi$ is at least quasi-concave.

We start our analysis by proving the following basic result.

Proposition 1 Under A1-A4 and for all $\alpha \in[0,1], \Omega_{\alpha}$ is a convex and compact set with $0 \in \Omega_{\alpha}$ and $\Omega_{1}=\mathcal{A}$.

Proof That $0 \in \Omega_{\alpha}$ and $\Omega_{1}=\mathcal{A}$ follow directly from (1)-(3). For $\alpha=1$, convexity holds trivially because $\Omega_{\alpha}=\mathcal{A}$ is convex. So let $\alpha \in\left[0,1\left[\right.\right.$. If $\Omega_{\alpha}$ contains only 
a single element, it is trivially convex. Otherwise, choose $a_{1}, a_{2} \in \Omega_{\alpha}$ and $\lambda \in[0,1]$. Let $a_{\lambda}:=\lambda a_{1}+(1-\lambda) a_{2}$, which is an element of $\mathcal{A}$, since $\mathcal{A}$ is convex. Consider $z_{1} \notin \Sigma\left(a_{1}\right)$ and $z_{2} \notin \Sigma\left(a_{2}\right)$, which exist due to $\alpha<1$, and let $z_{\lambda}:=\lambda z_{1}+(1-\lambda) z_{2}$. By A3, $z_{\lambda} \in \mathcal{Z}$. A2 implies that $\left(a_{\lambda}, z_{\lambda}\right) \succeq\left(0, z_{\lambda}\right)$, so that $z_{\lambda} \notin \Sigma\left(a_{\lambda}\right)$.

Let $\bar{\Sigma}(a)$ denote the complement of $\Sigma(a)$ in $\mathcal{Z}$ for some $a \in \mathcal{A}$. By using all combinations of $z_{1} \in \bar{\Sigma}\left(a_{1}\right)$ and $z_{2} \in \bar{\Sigma}\left(a_{2}\right)$, we can define $\bar{\Sigma}_{\lambda}:=\left\{\lambda z_{1}+(1-\right.$ $\left.\lambda) z_{2} \mid z_{1} \in \bar{\Sigma}\left(a_{1}\right), z_{2} \in \bar{\Sigma}\left(a_{2}\right)\right\}$ and get $\bar{\Sigma}_{\lambda} \subseteq \bar{\Sigma}\left(a_{\lambda}\right)$.

Now let $\bar{z}$ be a point of $\bar{\Sigma}\left(a_{1}\right) \cup \bar{\Sigma}\left(a_{2}\right)$ where $\phi(z)$ attains its maximum on this set. Assume without loss of generality that $\bar{z} \in \bar{\Sigma}\left(a_{1}\right)$. By A4, all points $\lambda \bar{z}+(1-\lambda) \tilde{z}$ are an element of all level sets that contain $\tilde{z}$. By choosing subsequently all $\tilde{z} \in \bar{\Sigma}\left(a_{2}\right)$, we thus get a set of points $H:=\left\{\lambda \bar{z}+(1-\lambda) \tilde{z} \mid \tilde{z} \in \bar{\Sigma}\left(a_{2}\right)\right\}$ with $P(H) \geq P\left(\bar{\Sigma}\left(a_{2}\right)\right) \geq 1-\alpha$. By construction, $H \subseteq \bar{\Sigma}_{\lambda}$. Therefore, $P\left(\bar{\Sigma}_{\lambda}\right) \geq P\left(\bar{\Sigma}\left(a_{2}\right)\right) \geq 1-\alpha$. So $P\left(\Sigma\left(a_{\lambda}\right)\right) \leq \alpha$ and thus $a_{\lambda} \in \Omega_{\alpha}$.

The compactness of $\Omega_{\alpha}$ follows from (3), $P$ being continuous and $\mathcal{A}$ being bounded.

Proposition 1 reveals an important property of the above definition of sustainability. If two actions are sustainable at some level $\alpha$, then all combinations of these actions are also sustainable at $\alpha$. Especially, if an action $a$ is sustainable at some $\alpha \in[0,1]$, then a downscaled action $\lambda a$ with $0 \leq \lambda<1$ is also sustainable at $\alpha$.

Under the assumption $\mathrm{A} 4 *$, we get the stronger result that combining or downscaling actions actually increases the level of sustainability. By assuming A4**, we gain information about the sustainability of a convex combination of actions that are sustainable at different levels.

Proposition 2 Assume $A 1-A 3$ and A4*. Let $a_{1}, a_{2} \in \Omega_{\alpha}$ with $\alpha>0$. Then for all $\lambda \in] 0,1\left[\right.$, we have $\lambda a_{1}+(1-\lambda) a_{2} \in \Omega_{\tilde{\alpha}}$ with $\tilde{\alpha}<\alpha$.

Assume A1-A3 and $A 4 * *$ and let $a_{1} \in \Omega_{\alpha_{1}}$ and $a_{2} \in \Omega_{\alpha_{2}}$. Then, for all $\lambda \in[0,1]$, we have $\lambda a_{1}+(1-\lambda) a_{2} \in \Omega_{\lambda \alpha_{1}+(1-\lambda) \alpha_{2}}$.

Proof The proof proceeds similarly to that of Proposition 1. The difference is that under A4*, we get $P(H)>P\left(\bar{\Sigma}\left(a_{2}\right)\right) \geq 1-\alpha$, because for every point of $\bar{\Sigma}\left(a_{2}\right)$, the corresponding point of $H$ lies in a higher level set. So we have $P\left(\bar{\Sigma}_{\lambda}\right)>P\left(\bar{\Sigma}\left(a_{2}\right)\right) \geq$ $1-\alpha$ and thus the assertion follows. Under A4**, we can assure $P(H) \geq \lambda P\left(\bar{\Sigma}\left(a_{1}\right)\right)+$ $(1-\lambda) P\left(\bar{\Sigma}\left(a_{2}\right)\right)$, so that $\lambda a_{1}+(1-\lambda) a_{2} \in \Omega_{\lambda \alpha_{1}+(1-\lambda) \alpha_{2}}$.

Proposition 2 is remarkable because assumption A $4 *$ is met by distributions that are frequently used to model expectations, like the multivariate normal distribution. Thus, under widely used modeling assumptions, a linear combination of two sustainable actions results in a lower risk of harming the future individual than each of the two original actions. This indicates a connection to portfolio choice, where diversification is used as a means to reduce risks. We elaborate on this in Sect. 5.

The part of Proposition 2 that holds under A4** is important because it provides information concerning how the achievable level of sustainability changes if we combine two actions. This result will be helpful for characterizing the trade-off between sustainability and efficiency in Sect. 4.

The final result of this section provides some further information about the above concept of sustainability. 
Proposition 3 Under $A 1-A 4$, we have $\Omega_{\alpha_{1}} \subseteq \Omega_{\alpha_{2}}$ for $\alpha_{1} \leq \alpha_{2}$. If in addition A4* holds, then $\Omega_{\alpha}:[0,1] \rightarrow \mathcal{A}$ is a continuous map of $\alpha$.

Proof $\Omega_{\alpha_{1}} \subseteq \Omega_{\alpha_{2}}$ for $\alpha_{1} \leq \alpha_{2}$ is a consequence of (1)-(3), because if $a \in \Omega_{\alpha}$, then $a \in \Omega_{\tilde{\alpha}}, \forall \tilde{\alpha} \geq \alpha$.

Let $a \in \operatorname{int}\left(\Omega_{\alpha}\right)$, which implies that there exists an $\varepsilon>0$, so that $a+\varepsilon \in \Omega_{\alpha}$ and $a-\varepsilon \in \Omega_{\alpha}$. Under A4*, Proposition 2 assures that $a_{1}:=(1 / 2) a+(1 / 2)(a-\varepsilon) \in \Omega_{\tilde{\alpha}}$ and $a_{2}:=(1 / 2) a+(1 / 2)(a+\varepsilon) \in \Omega_{\tilde{\alpha}}$ with some $\tilde{\alpha}<\alpha$. By Proposition $1, \Omega_{\tilde{\alpha}}$ is convex, so that $a=(1 / 2) a_{1}+(1 / 2) a_{2} \in \Omega_{\tilde{\alpha}}$. So if $a \in \operatorname{int}\left(\Omega_{\alpha}\right)$, then there exists an $\tilde{\alpha}<\alpha$ with $a \in \Omega_{\tilde{\alpha}}$. Since $\Omega_{\alpha_{1}} \subseteq \Omega_{\alpha_{2}}$ for $\alpha_{1} \leq \alpha_{2}$, we trivially have $a \in \operatorname{int}\left(\Omega_{\alpha}\right) \Rightarrow a \in \Omega_{\tilde{\alpha}}$ for $\tilde{\alpha}>\alpha$. So given that by Proposition $1, \Omega_{\alpha}$ is a closed set for all $\alpha \in[0,1], \Omega_{\alpha}:[0,1] \rightarrow \mathcal{A}$ is continuous map.

Proposition 3 shows that small changes to the level of sustainability $\alpha$ result only in small changes to the set of sustainable actions. Thus, there are no thresholds at which slightly altering $\alpha$ enforces drastic changes to hitherto sustainable actions.

So far, our analysis has shown that the concept of sustainability advanced in Krysiak and Krysiak (2006) provides a mathematically convenient framework for analyzing questions of intergenerational distribution. The compactness, convexity, and continuity properties proven above assure that the concept integrates easily into optimization frameworks and thus into economic analysis.

\section{Sustainability and efficiency}

In analogy to the relation between type I and type II errors in statistical test theory, we can expect that reducing the risk of harming a future individual increases the probability of foregoing actions that would have benefited everyone. So, there might be a trade-off between sustainability and efficiency.

In models with uncertainty, two efficiency concepts, namely, ex ante and ex post efficiency are commonly used. In our context, neither of these concepts is directly useful. Ex ante efficiency is based on expected outcomes, whereas we use a definition of sustainability that is based on actual outcomes. Thus, ex ante efficiency corresponds to a different ethical view on intergenerational risk sharing than our sustainability concept. Consequently, comparing these concepts may be mathematically feasible, ${ }^{3}$ but it is conceptually uninteresting, because comparing two normative concepts by using versions of these concepts that are based on fundamentally different premises does not yield reliable information about the compatibility of the concepts in general. Furthermore, in many applications, an interesting question is whether "protecting" future generations reduces the likelihood that projects are pursued would have increased present and future welfare. To address this question, an ex post evaluation of actions is necessary, which rules out ex ante efficiency.

\footnotetext{
3 Indeed, Propositions 1 and 3 guarantee that, under A1-A3 and A4*, there exists a minimal level of $\alpha$ for which an ex ante efficient action is sustainable (By Proposition 1, all ex ante efficient actions are sustainable for $\alpha=1$ and the continuity proven in Proposition 3 implies that there is a lowest value of $\alpha$ in $[0,1]$ for which at least one ex ante efficient action is an element of $\Omega_{\alpha}$ ). Thus, ex ante efficiency and our notion of sustainability are compatible, whenever the sustainability requirement is not too strict.
} 
The notion of ex post efficiency is more natural in our context; being based on actual outcomes, it is conceptually similar to the definition of sustainability used here. However, in our setup, actions have to be chosen under informational constraints. Thus, it is unlikely that there exists an action that is ex post efficient with certainty. Therefore, we use a weakened concept of ex post efficiency by considering the probability that an action leads to an ex post efficient outcome. This results in a concept of efficiency that is closely related to the above definition of sustainability.

To characterize efficiency in our model, we need information concerning present preferences. We assume that present preferences are certain and can be depicted by the above-defined "master" preferences for $t=t_{0} \in \mathcal{T}$. Furthermore, we assume that there is no uncertainty w.r.t. the present outcomes of an action. The present state of nature shall be $s_{0} \in \mathcal{S}$. For notational simplicity, we set $z_{0}:=\left(s_{0}, t_{0}\right)$.

We define the probability that an action is ex post Pareto inferior to another action by

$$
\xi(a):=P(\tilde{\Sigma}(a))
$$

with

$$
\begin{aligned}
\tilde{\Sigma}(a):= & \left\{z \in \mathcal{Z} \mid \exists b \in \mathcal{A} \text { with }\left((b, z) \succ(a, z) \wedge\left(b, z_{0}\right) \succeq\left(a, z_{0}\right)\right)\right. \\
& \left.\vee\left((b, z) \succeq(a, z) \wedge\left(b, z_{0}\right) \succ\left(a, z_{0}\right)\right)\right\} .
\end{aligned}
$$

So, $\xi(a)$ measures the probability that an action $a$ is ex post Pareto dominated by some other action $b$. As in (3), we can define level sets of $\mathcal{A}$ w.r.t. to this measure.

$$
\Xi_{\beta}:=\{a \in \mathcal{A} \mid \xi(a) \leq \beta\}
$$

Thus, $\Xi_{\beta}$ is the set of actions whose probability of being ex post inefficient is at most $\beta$. Note that in contrast to $\Omega_{\alpha}$, which is non-empty for all $\alpha \in[0,1]$, it is possible that $\Xi_{\beta}=\emptyset$ for some $\beta \in[0,1]$.

As in the preceding section, our assumptions assure that this set has convenient properties.

Proposition 4 Assume A1-A4. Then, there exists a $\beta_{\min } \in[0,1]$ so that for all $\beta \geq \beta_{\text {min }}$, we have $\Xi_{\beta} \neq \emptyset$, and for all $\beta<\beta_{\text {min }}$, we have $\Xi_{\beta}=\emptyset$. Furthermore, $\Xi_{\beta}$ is a convex and compact set for all $\beta \in\left[\beta_{\text {min }}, 1\right]$ and we have $\Xi_{\beta_{1}} \subseteq \Xi_{\beta_{2}}$ for $\beta_{1} \leq \beta_{2}$ as well as $\Xi_{1}=\mathcal{A}$.

If in addition $A 4 *$ holds, then $a_{1}, a_{2} \in \boldsymbol{\Xi}_{\beta}$ with $\beta>\beta_{\text {min }}$ implies $\lambda a_{1}+(1-\lambda) a_{2} \in$ $\Xi_{\tilde{\beta}}$ with $\tilde{\beta}<\beta$, for all $\left.\lambda \in\right] 0,1\left[\right.$, as well as that $\Xi_{\beta}:\left[\beta_{\text {min }}, 1\right] \rightarrow \mathcal{A}$ is a continuous function of $\beta$.

If $A 1-A 3$ and $A 4 * *$ hold, then $a_{1} \in \Xi_{\beta_{1}}$ and $a_{2} \in \Xi_{\beta_{2}}$ imply $\lambda a_{1}+(1-\lambda) a_{2} \in$ $\Xi_{\lambda \beta_{1}+(1-\lambda) \beta_{2}}$ for all $\lambda \in[0,1]$.

Proof By (4) and (5), $\xi(a) \in[0,1]$ is a continuous function of $a \in \mathcal{A}$ because $\mathcal{A}$ is convex and $P$ as well as $\succ$ are continuous. So, $\xi(a)$ attains its minimum on the compact set $\mathcal{A}$. This minimum equals $\beta_{\min }$ : For $\beta<\beta_{\min }$, we have $\Xi_{\beta}=\emptyset$, for $\beta=\beta_{\min }$, the 
definition of $\beta_{\min }$ assures that there is at least one $a \in \Xi_{\beta}$, so that $\Xi_{\beta} \neq \emptyset$, and for $\beta>\beta_{\min }, \Xi_{\beta_{\min }} \subseteq \Xi_{\beta}$, which directly results from (6), implies $\Xi_{\beta} \neq \emptyset$.

Compactness follows from (6) and $\mathcal{A}$ being bounded. For $\beta=1$, convexity is trivial because $\Xi_{1}=\mathcal{A}$ is convex. For $\beta \in\left[\beta_{\min }, 1\right.$ [, we can find $z_{1} \notin \tilde{\Sigma}\left(a_{1}\right), z_{2} \notin \tilde{\Sigma}\left(a_{2}\right)$ for all $a_{1}, a_{2} \in \Xi_{\beta}$ (if $\Xi_{\beta}$ contains only a single element, we do not have to prove convexity; it holds trivially). By A2, we have $z_{\lambda} \notin \tilde{\Sigma}\left(a_{\lambda}\right)$ with $a_{\lambda}:=\lambda a_{1}+(1-\lambda) a_{2}$ and $z_{\lambda}:=\lambda z_{1}+(1-\lambda) z_{2}$ for all $\lambda \in[0,1]$. With this information, the proofs of Propositions $1-3$ can be transferred directly to yield the assertions.

Proposition 4 shows that the mathematical properties derived for the set of sustainable actions $\Omega_{\alpha}$ also hold for the set of actions for which the probability that they turn out to be ex post inefficient, is at most $\beta$.

To inquire in how far our definitions of sustainability and efficiency are compatible, we define

$$
\Gamma:=\left\{(\alpha, \beta) \in[0,1] \times[0,1] \mid \Omega_{\alpha} \cap \Xi_{\beta} \neq \emptyset\right\} .
$$

Thus, $\Gamma$ is the set of all combinations of $\alpha$ and $\beta$ that are simultaneously supported by at least one feasible action. To characterize this set, it is helpful to define its lower boundary:

$$
\beta^{*}(\alpha):=\min \left\{\beta \in[0,1] \mid \Omega_{\alpha} \cap \Xi_{\beta} \neq \emptyset\right\}
$$

The following proposition provides some information concerning $\Gamma$ and $\beta^{*}(\alpha)$.

Proposition 5 Assume A1-A4. Then, $\Gamma$ is compact. Furthermore, if $(\alpha, \beta) \in \Gamma$, then $(\tilde{\alpha}, \tilde{\beta}) \in \Gamma$ for all $\alpha \leq \tilde{\alpha} \leq 1, \beta \leq \tilde{\beta} \leq 1$. Also, $\beta^{*}(\alpha)$ is defined for all $\alpha \in[0,1]$ and is a decreasing function of $\alpha$ with $\beta^{*}(1)=\beta_{\min }$.

Under $A 1-A 3$ and $A 4^{* *}, \Gamma$ is in addition a convex set.

Under $A 1-A 3$ and $A 4^{*}, \beta^{*}(\alpha):[0,1] \rightarrow[0,1]$ is in addition a continuous function and is strictly decreasing in $\alpha$ for all $\alpha \in[0,1]$ with $\beta^{*}(\alpha)>\beta_{\min }$.

Proof By construction, $\Gamma$ is bounded. Let $\alpha \in[0,1]$ be given. By Proposition $1, \Omega_{\alpha}$ is convex and compact, so that the continuous function $\xi(a)$ attains its minimum on $\Omega_{\alpha}$. Thus, for a given $\alpha$, the set of $\beta$ with $(\alpha, \beta) \in \Gamma$ is closed. Since $\alpha \in[0,1], \Gamma$ is also closed and thus compact. Furthermore, due to $\Xi_{1}=\mathcal{A}$, there is always a $\beta \in[0,1]$ with $\Omega_{\alpha} \cap \Xi_{\beta} \neq \varnothing$. So given the continuity of $\xi(a)$ and the compactness of $\Gamma, \beta^{*}(\alpha)$ is well defined for all $\alpha \in[0,1]$.

$(\alpha, \beta) \in \Gamma \Rightarrow(\tilde{\alpha}, \tilde{\beta}) \in \Gamma$ for all $\tilde{\alpha} \geq \alpha, \tilde{\beta} \geq \beta$ follows from $\Xi_{\beta_{1}} \subseteq \Xi_{\beta_{2}}$, for $\beta_{1} \leq \beta_{2}$, and $\Omega_{\alpha_{1}} \subseteq \Omega_{\alpha_{2}}$, for $\alpha_{1} \leq \alpha_{2}$ (Propositions 3 and 4 ).

If $\left(\alpha_{1}, \beta_{1}\right),\left(\alpha_{2}, \beta_{2}\right) \in \Gamma$, then there exist $a_{1}, a_{2}$ with $a_{1} \in \Omega_{\alpha_{1}} \cap \Xi_{\beta_{1}}$ and $a_{2} \in$ $\Omega_{\alpha_{2}} \cap \Xi_{\beta_{2}}$. Under A4**, Propositions 2 and 4 imply $\lambda a_{1}+(1-\lambda) a_{2} \in \Omega_{\lambda \alpha_{1}+(1-\lambda) \alpha_{2}}$ and $\lambda a_{1}+(1-\lambda) a_{2} \in \Xi_{\lambda \beta_{1}+(1-\lambda) \beta_{2}}$, so that $\Omega_{\lambda \alpha_{1}+(1-\lambda) \alpha_{2}} \cap \Xi_{\lambda \beta_{1}+(1-\lambda) \beta_{2}} \neq \emptyset$ and therefore $\left(\lambda \alpha_{1}+(1-\lambda) \alpha_{2}, \lambda \beta_{1}+(1-\lambda) \beta_{2}\right) \in \Gamma$. So under A4**, $\Gamma$ is convex.

By Proposition 3, we have $\Omega_{\alpha_{1}} \subseteq \Omega_{\alpha_{2}}$ for $\alpha_{1} \leq \alpha_{2}$, so that $\beta^{*}(\alpha)$ is decreasing in $\alpha$. Since $\Omega_{1}=\mathcal{A}$, we have $\beta^{*}(1)=\beta_{\min }$ by the definition of $\beta_{\min }$. 
Under A4*, $\beta^{*}(\alpha)$ is a continuous function, because by Propositions 3 and $4, \Omega_{\alpha}$ and $\Xi_{\beta}$ are continuous maps and $\xi(a)$ is a continuous function.

Finally under A4*, $\Xi_{\beta^{*}(\alpha)}$ contains strictly more than a single element for all $\alpha \in[0,1]$ with $\beta^{*}(\alpha)>\beta_{\min }$ (otherwise $\Xi_{\tilde{\beta}}$ would be empty for all $\tilde{\beta}<\beta^{*}(\alpha)$ contradicting $\beta^{*}(\alpha)>\beta_{\min }$ ). By Proposition 6 below (which is independent from the current proof), only one of these elements can also be an element of $\Omega_{\alpha}$. So there exists an $\tilde{a} \in \Xi_{\beta^{*}(\alpha)}$ with $\tilde{a} \notin \Omega_{\alpha}$ but $\tilde{a} \in \Omega_{\tilde{\alpha}}$ for some $\tilde{\alpha}>\alpha$. By the definition of $\beta^{*}(\alpha)$, there also exists an $a \in \Xi_{\beta^{*}(\alpha)} \cap \Omega_{\alpha}$, which (by Proposition 4 and the definition of $\beta^{*}(\alpha)$ ) must be an element of the boundary of $\Omega_{\alpha}$. Therefore, $a_{\lambda}:=\lambda a+(1-\lambda) \tilde{a} \notin \Omega_{\alpha}$ for all $\lambda \in] 0,1$ [. Since $a_{\lambda} \in \Omega_{\tilde{\alpha}}$, the continuity of $\Omega_{\alpha}$ as a function of $\alpha$ assures that for all $\left.\alpha_{\lambda} \in\right] \alpha, \tilde{\alpha}[$, there is a $\lambda \in] 0,1\left[\right.$ so that $a_{\lambda} \in \Omega_{\alpha_{\lambda}}$. By construction, $a_{\lambda} \in \operatorname{int}\left(\Xi_{\beta^{*}(\alpha)}\right)$ for all $\lambda \in] 0,1\left[\right.$. Thus by Proposition 4, there exists a $\beta_{\lambda}<\beta^{*}(\alpha)$ so that $a_{\lambda} \in \Xi_{\beta_{\lambda}}$. In conclusion, if $\beta^{*}(\alpha)>\beta_{\min }$, then there exists an $\varepsilon>0$, so that for all $\left.\left.\alpha_{\lambda} \in\right] \alpha, \alpha+\varepsilon\right]$, there is a $\beta_{\lambda}<\beta^{*}(\alpha)$ with $\left(\alpha_{\lambda}, \beta_{\lambda}\right) \in \Gamma$. Consequently, $\beta^{*}(\alpha)$ is strictly decreasing in $\alpha$.

Proposition 5 has several important implications. There is a monotonic relation between the probability of harming future individuals and the probability of not pursuing actions that would benefit everyone. Under A4*, this relation is even strictly monotonic until the minimal achievable level of $\beta$ is reached: demanding more sustainability implies that we get less efficiency. Since we have imposed no restrictions on the set of possible actions or on present or future preferences that could explain this trade-off, this result indicates that there is an inherent incompatibility of efficiency and sustainability.

Under A4*, this trade-off has a convenient continuity property: small changes to the level of sustainability result only in small changes to the probability of efficiency. Finally, under A4**, the set $\Gamma$ is convex, implying that increasing requirements of sustainability necessitate non-decreasing marginal sacrifices in terms of efficiency.

It is possible to use the above results to characterize a special set of actions, which we refer to as "most efficient sustainable actions" (MESA):

$$
\text { MESA: } a^{*}(\alpha):=\left\{a \in \Omega_{\alpha} \cap \Xi_{\beta^{*}(\alpha)}\right\} \text {. }
$$

In the concept of a "most efficient sustainable action," we first limit the risk of harming future individuals by setting $\alpha$ and then choose those actions for which the risk that they turn out to be ex post inefficient is minimized. In other words, a MESA is an action for which the type I error (allowing an action that harms the future individual) is constrained and the type II error (rejecting an action that is beneficial for all) is minimized under this constraint. This is similar to Woodward (2000), where sustainability is used as a constraint in a welfare maximization.

The following proposition characterizes these actions.

Proposition 6 Under $A 1-A 3$ and $A^{*}, a^{*}(\alpha):[0,1] \rightarrow \mathcal{A}$ is well defined for all $\alpha \in[0,1]$ and is a single-valued, continuous function of $\alpha$.

Proof Due to $0 \in \Omega_{\alpha} \forall \alpha \in[0,1]$ and by the definition of $\beta^{*}(\alpha)$, the set $a^{*}(\alpha)$ is non-empty $\forall \alpha \in[0,1]$, so that $a^{*}(\alpha)$ is well defined. 
Now assume that $a^{*}(\alpha)$ would be a set with strictly more than one element. Then we would have $a_{1}, a_{2} \in a^{*}(\alpha)$ and by Propositions 1 and 4 , we would have $a_{\lambda}:=$ $\lambda a_{1}+(1-\lambda) a_{2} \in a^{*}(\alpha)$. By Proposition $4, a_{\lambda} \in \Xi_{\tilde{\beta}}$ with $\tilde{\beta}<\beta^{*}(\alpha)$ for $\left.\lambda \in\right] 0,1[$, which contradicts the definition of $\beta^{*}(\alpha)$. Therefore, $a^{*}(\alpha)$ has to be single valued. The continuity of $a^{*}(\alpha)$ follows from that of $\Omega_{\alpha}, \Xi_{\beta}$, and $\beta^{*}(\alpha)$.

Proposition 6 shows that defining "most efficient sustainable actions" is feasible for all $\alpha \in[0,1]$ and that this concept identifies exactly one action for every value of $\alpha$. Furthermore, small changes to the level of sustainability induce only small changes to the "most efficient sustainable action."

We could also first set a probability of achieving ex post efficiency and then minimize the risk of harming the future individual under this constraint. This would result in a function $\bar{a}^{*}(\beta)$. As can be easily shown, this is simply a dual representation of $a^{*}(\alpha)$. For $\beta \in\left[\beta_{\min }, 1\right]$, a so defined function has properties that closely resemble the above proven ones of $a^{*}(\alpha)$.

Altogether, the analysis of this section has shown that there is an inherent trade-off between sustainability and ex post efficiency and that this conflict can be conveniently formalized and characterized in a risk-based framework. Indeed, the uncertainty is helpful in this context in that it allows for a smooth transition between an efficiency-focused and a distribution-centered approach. The trade-off between efficiency and sustainability can be minimized by considering only "most efficient sustainable actions," which is an economically intuitive and, by Proposition 6, also a mathematically convenient choice rule.

\section{Sustainability through Diversification}

Propositions 2 and 4 have shown that diversification, in the sense of combining actions, reduces the risk of being unsustainable as well as that of being ex post inefficient. This indicates a connection to portfolio theory, where diversification is used to reduce the overall risk of a portfolio.

In this section, we explore the connection of our framework to mean-variance analysis. We have chosen this particular concept of portfolio theory because it yields an interesting connection to our concept and mean-variance analysis is increasingly used in environmental economics to address problems of designing or evaluating policies with long-lasting consequences. For example, Baldursson and Magnusson (1997) and Edwards et al. (2004) used mean-variance analysis in the context of fishery management, and Springer (2003) analyzed the question in how far investments into climate change mitigation should be diversified across countries with a mean-variance approach.

These studies directly employ a mean-variance criterion for policy evaluation without formally addressing the question of how such a criterion can be justified in the normative context of policy evaluation. We inquire whether a risk-based sustainability concept can provide a theoretical foundation for such applications by instating a connection between policy evaluation and portfolio choice.

We establish such a connection albeit only under restrictive assumptions on preference and outcome uncertainty. These assumptions are frequently used in 
mean-variance analysis but have been empirically refuted in many applications in finance. As we discuss below, they are also disputable in our context, except for a special case.

We need two additional assumptions. These are most easily stated in terms of utility, which is possible, because A1 implies the existence of a utility function $u(a, z)$ and thus of a utility difference $\Delta u(a, z):=u(a, z)-u(0, z)$. Note that this difference does not include a utility comparison between types but only between actions.

A5 For all $a \in \mathcal{A}, \Delta u(a, z)$ is a normally distributed random variable with expectation $\mu(a)$ and variance $\sigma^{2}(a)$ that both depend continuously on $a \in \mathcal{A}$.

A6 If $\mu\left(a_{1}\right) \geq \mu\left(a_{2}\right)$, then $u\left(a_{1}, z_{0}\right) \geq u\left(a_{2}, z_{0}\right)$, and vice versa.

A5 imposes a specific functional form on the probability of future types and situations. It implies A4*, but it is not compatible with A4**. As Samuelson (1970) has shown, A5 holds approximately, if the uncertainty is sufficiently small.

Assumption A6 implies that an increase in expected future benefits is also beneficial for the present generation, and vice versa. It resembles a scenario where the probability that future conditions and preferences diverge only slightly from present ones is not too small and where there is no genuine conflict of interest, that is, where presently beneficial actions are also in the interest of the future individual, if its situation and its preferences are close to present ones. This assumption excludes deterministic distributional conflicts. In Sect. 6, we discuss how such conflicts can be reintegrated.

Note that by A5, the influence of the variance $\sigma^{2}(a)$ on the risk of harming the future individual depends on $\mu(a)$ : for a constant $\mu(a)>0$, this risk is increasing in $\sigma^{2}(a)$, whereas for a constant $\mu(a)<0$, this risk is decreasing in $\sigma^{2}(a)$. To avoid case distinctions, we specialize to the case $\mu(a) \geq 0$. Since under A5, it is necessary to have $\mu(a) \geq 0$ for $a \in \Omega_{0.5}$ and since a risk of more than $50 \%$ of harming future individuals is hardly compatible with an ethically tenable notion of sustainability, this restriction seems to be a reasonable simplification.

We are interested in establishing a connection between the above risk-based concept of sustainability and the efficient frontier of mean-variance analysis. The efficient frontier is the minimal variance $\sigma^{2}(a)$ achievable for a given expected value $\mu(a)$ :

$$
\sigma_{\mathrm{eff}}^{2}(\mu):=\min _{a \in \mathcal{A}} \sigma^{2}(a) \quad \text { with } \mu(a)=\mu \text {. }
$$

Usually, $\sigma_{\text {eff }}^{2}$ will have up- and downward sloping parts. But for our analysis, only the strictly upward sloping parts are relevant. These are given by

$$
\Psi:=\left\{\left(\mu, \sigma^{2}\right) \in \mathbb{R} \times \mathbb{R}_{+} \mid \mu=\max \sigma_{\mathrm{eff}}^{2-1}\left(\sigma^{2}\right)\right\}
$$

where $\sigma_{\text {eff }}^{2-1}\left(\sigma^{2}\right)$ denotes the (usually set-valued and possibly empty) pre-image of $\sigma_{\text {eff }}^{2}(\mu)$ for $\sigma_{\text {eff }}^{2}=\sigma^{2}$. We refer to actions that lead to points on the upward sloping part of the efficient frontier as "efficient frontier actions" (EFA).

$$
\mathrm{EFA}: \mathcal{A}^{\times}:=\left\{a \in \mathcal{A} \mid\left(\mu(a), \sigma^{2}(a)\right) \in \Psi\right\}
$$


The following proposition connects these "efficient frontier actions" with the "most efficient sustainable actions" introduced in the preceding section.

Proposition 7 Assume A1-A3, A4*, and A5-A6. Then, the set $\mathcal{A}^{*}:=\left\{a^{*}(\alpha) \mid 0 \leq \alpha \leq\right.$ $0.5\}$ of "most efficient sustainable actions" with $\alpha \leq 0.5$ is a compact and connected subset of the set $\mathcal{A}^{\times} \cap\{a \in \mathcal{A} \mid \mu(a) \geq 0\}$ of "efficient frontier actions" with $\mu(a) \geq 0$.

Proof Assume that there is an $a_{1} \in \mathcal{A}$ with $a_{1} \in a^{*}(\alpha)$, for some $\alpha \in[0,0.5]$, but $a_{1} \notin \mathcal{A}^{\times}$. Then, there exists an $a_{2} \in \mathcal{A}$ with $\mu\left(a_{2}\right)>\mu\left(a_{1}\right)$ and $\sigma^{2}\left(a_{2}\right)=\sigma^{2}\left(a_{1}\right)$. But by A5 and A6, $\sigma^{2}\left(a_{2}\right)=\sigma^{2}\left(a_{1}\right)$ and $\mu\left(a_{2}\right)>\mu\left(a_{1}\right)$ imply that if $a_{1} \in \Omega_{\alpha} \cap \Xi_{\beta}$, then $a_{2} \in \Omega_{\tilde{\alpha}} \cap \Xi_{\beta}$ with $\tilde{\alpha}<\alpha$. Therefore, $a_{1} \in a^{*}(\alpha)$ is contradicted. So, $a \in a^{*}(\alpha)$ implies $a \in \mathcal{A}^{\times}$. Thus every MESA is an EFA and since by A5, $\mu(a) \geq 0$ is necessary for $a \in \Omega_{0.5}$, we have $\mathcal{A}^{*}:=\left\{a^{*}(\alpha) \mid 0 \leq \alpha \leq 0.5\right\} \subseteq \mathcal{A}^{\times} \cap\{a \in \mathcal{A} \mid \mu(a) \geq 0\}$. Furthermore, by Proposition $6, a^{*}(\alpha)$ is a continuous function of $\alpha$. Therefore $\mathcal{A}^{*}$, being the image of the compact and connected interval $[0,0.5]$ under $a^{*}(\alpha)$, is a compact and connected set.

Proposition 7 indicates a link between sustainability under uncertainty and portfolio theory. This implies that we can transfer results from mean-variance analysis (see, e.g., Markowitz and Todd 1987) to the context of policy evaluation and that a riskbased concept of sustainability provides a theoretical basis on which mean-variance analysis can be used for policy evaluation. In addition, it is possible to derive a set of candidates for "most efficient sustainable actions" by calculating the efficient frontier.

As noted above, Assumptions A5 and A6 are highly restrictive. Whereas Assumption A6 can be relaxed (see Sect. 6), Assumption A5 is crucial for the above result. As shown by Samuelson (1970), it can be seen as an approximation for low levels of uncertainty. But in other cases, it is questionable whether future welfare effects of present actions are normally distributed. Furthermore, this question is not easily answered empirically because it refers to expectations over possible future states. ${ }^{4}$ Thus, applying Proposition 7 will mainly be possible in cases where the uncertainty is sufficiently small, so that A5 holds approximately.

\section{Limitations and extensions}

We have intentionally constrained our analysis to a simple setup that contains only one present and one future individual. This framework has facilitated a clear focus on the problem of distributing risks between individuals. But it deviates from the setup in which sustainability is commonly discussed, which consists of a sequence of (possibly overlapping) generations. Whether our analysis can be extended to such a setup is thus an important question. Also, we have excluded deterministic distributional problems from the analysis in Sect. 5 and have not addressed the question of whether our assumptions on preferences and expectations can be relaxed. In this section, we briefly discuss these points.

Consider a number $N$ of future individuals, possibly living in different periods, and let $\phi\left(z_{1}, \ldots, z_{N}\right)$ be a density that characterizes the likelihood of a particular

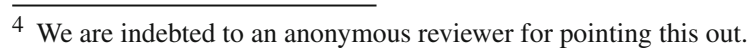


assignment of types and states of natures to these individuals and the times at which they live. We can define $\Omega_{\alpha}$ as the set of actions for which the probability that at least one of the $N$ individuals is harmed, is at most $\alpha$. Similarly, the set $\Xi_{\beta}$ can be defined as the set of actions for which the probability that they turn out to be Pareto dominated according to the preferences of the present and all $N$ future individuals is limited by $\beta$. With some slight adjustments in stating our assumptions, the results of the preceding sections can be directly transferred to this setup. This framework encompasses a broad range of time structures, if we allow for correlations in $\phi\left(z_{1}, \ldots, z_{N}\right)$. The state of nature and the types of individuals living in temporal proximity can be more strongly correlated than those of individuals living in different millenia. This covers setups with sequentially living generations and with overlapping generations.

We can also relax Assumption A6, that is, the exclusion of deterministic conflicts of interest between future and present individuals, by viewing decisions as a twostep process. In a first step, combinations of actions are calculated that assure that each of these combinations does not harm the present whenever it does not decrease expected future welfare, and vice versa. For example, in a typical resource-use-andinvestment-type problem, we can combine resource use, which benefits the present (possibly) at the cost of the future, with a sufficient investment in capital stocks, which benefits the future at the cost of the present. So, this first step handles deterministic distribution conflicts. In a second step, we use the thus defined combined actions as the set $\mathcal{A}$ and employ the methods advanced in the preceding sections to handle the uncertainty of future preferences and outcomes. In this way, we can combine a "conventional" sustainability analysis that proceeds under certainty with the above risk-based approach.

In contrast, Assumptions $\mathrm{A} 1-\mathrm{A} 4$ as well as the convexity of $\mathcal{A}$ are essential for our analysis. Most of our results hinge on the convexity of $\Omega_{\alpha}$ and $\Xi_{\beta}$ proven in Propositions 1 and 4, respectively. If $\mathcal{A}$ or $\mathcal{Z}$ are not convex, then $\Omega_{\alpha}$ and $\Xi_{\beta}$ will not be convex in general. For $\mathcal{A}$, this is obvious because $\Omega_{1}, \Xi_{1}=\mathcal{A}$. For $\mathcal{Z}$ there is some leeway. If, e.g., $\mathcal{Z}$ consists only of two elements $z_{1}, z_{2}$ with probabilities $p_{1}$ and $\left(1-p_{1}\right)$, with $p_{1}>\left(1-p_{1}\right)$, then $\Omega_{\alpha}$ and $\Xi_{\beta}$ will be convex for $\alpha, \beta<p_{1}$ if preferences are convex w.r.t. $a .^{5}$ Thus, most of our results can be transferred to such a setup: A 3 and A4 can be replaced by the convexity of preferences, A4* requires preferences to be strictly convex, only the results based on A4** will have no direct counterparts. However, for more general non-convex sets $\mathcal{Z}$, it is often not possible to transfer our results. For example, it is easy to find a set $\mathcal{Z}$ that consists of three elements, so that $\Omega_{\alpha}$ and $\Xi_{\beta}$ are not convex. ${ }^{6}$ Thus, although there are cases in which most of our results hold without $\mathcal{Z}$ being convex, these seem to be special cases.

\footnotetext{
5 If $\alpha$ is smaller than $p_{1}$, we cannot have $z_{1} \in \Sigma(a)$ for any $a \in \Omega_{\alpha}$. So if $a_{1}, a_{2} \in \Omega_{\alpha}$, then $z_{1} \notin \Sigma\left(a_{1}\right)$ and $z_{1} \notin \Sigma\left(a_{2}\right)$. By the convexity of preferences w.r.t. $a$, we have $\left(\lambda a_{1}+(1-\lambda) a_{2}, z_{1}\right) \succeq\left(a_{1}, z_{1}\right)$ and thus $\lambda a_{1}+(1-\lambda) a_{2} \in \Omega_{\alpha}$. The convexity of $\Xi_{\beta}$ can be shown similarly.

6 Consider the case, where $a_{1}, a_{2} \in \Omega_{\alpha}, z_{1}, z_{2} \in \Sigma\left(a_{1}\right), z_{3} \notin \Sigma\left(a_{1}\right), z_{1}, z_{3} \in \Sigma\left(a_{2}\right)$, and $z_{2} \notin \Sigma\left(a_{2}\right)$. If $\left(a_{1}, z_{3}\right) \succ\left(\lambda a_{1}+(1-\lambda) a_{2}, z_{2}\right)$ and $\left(a_{2}, z_{2}\right) \succ\left(\lambda a_{1}+(1-\lambda) a_{2}, z_{3}\right)$ for some $\left.\lambda \in\right] 0,1[$, which is possible even if preferences are convex w.r.t. $a$, we can have $\lambda a_{1}+(1-\lambda) a_{2} \notin \Omega_{\alpha}$.
} 
Assumption A4 is also essential for our analysis. If the distribution of future states of nature and types is not unimodal, it is easily possible to construct examples in which $\Omega_{\alpha}$ and $\Xi_{\beta}$ are not convex, so that our main results do not hold.

Altogether, the applicability of our results is not constrained by the two-person setup but rather by our convexity assumptions and the restrictions on the likelihood of future states and preferences. Whereas the convexity assumptions are standard in economic analysis, the restriction on the probability distribution are clearly debatable. But we think that they cover interesting cases. Often, it will be prudent to assume that future individuals and future circumstances are close to present ones, so that the likelihood of a future state-type combination decreases, the further this combination deviates from the presently observed one. This corresponds closely to the main idea behind the unimodality demanded in A4.

\section{A numerical example}

To illustrate our analysis, we consider a simple numerical example. Assume that the present individual can bequest two stocks of capital, produced capital $K$ and natural capital $N$, to the future individual. The possible actions are changes to the capital stocks with outcomes $x_{N}=\left(1-a_{N}\right) N_{0}$ and $x_{K}=s\left(1+a_{K}\right) K_{0}$, where $N_{0}$ and $K_{0}$ denote the original values of the capital stocks. The random variable $s$ describes an uncertain change to the stock of produced capital. For example, it could represent uncertainty w.r.t. to the efficiency of investments into man-made capital or an uncertain growth or depreciation rate of produced capital. We could also introduce uncertainty w.r.t. the change of the stock of natural capital. But this would only complicate the example without allowing for additional insights.

Let the preferences of the future individual be given by a linear utility function $U\left(x_{K}, x_{N}, t\right)=x_{K}+t x_{N}$. From the perspective of the present, the type $t$ of the future individual is a random variable. Furthermore, assume that $s$ and $t$ are independently normally distributed with $s \sim N\left(\mu_{s}, \sigma_{s}\right)$ and $t \sim N\left(\mu_{t}, \sigma_{t}\right)$. Finally, the set of feasible actions $\mathcal{A}$ shall be given by $\mathcal{A}=\left\{\left(a_{K}, a_{N}\right) \in[0,1] \times[0,1]\right\}$. Thus, any decline in natural capital is irreversible.

Note that the above assumptions comply with A1-A4, and A4*. So we can apply Propositions 1-6.

For the following numerical calculations, we use the following parameter values: $\mathcal{E}(s)=1.5, \operatorname{Var}(s)=1, \mathcal{E}(t)=1, \operatorname{Var}(t)=0.1$. With these settings, the set $\Omega_{\alpha}$ can be calculated and is depicted in Fig. 1 for different values of $\alpha$. As this figure shows, the sets of sustainable actions are convex and compact and are contained in each other for increasing values of $\alpha$, as Propositions 1-3 suggest.

We can also numerically characterize the trade-off between sustainability and efficiency. Given that with the above specification of $\mathcal{A}$, every action $\left(a_{K}, a_{N}\right) \in \mathcal{A}$ with $a_{K}<1$ and $a_{N}>0$, will be dominated by $(1,0)$ with a very high probability, we introduce the additional constraint $a_{K} \leq a_{N}$. This constraint limits the productivity of natural capital in producing physical capital, which seems to be a reasonable description of most production processes. Furthermore, we assume that present preferences are given by $t_{0}=\mathcal{E}(t)$ and that $s_{0}=\mathcal{E}(s)$. 


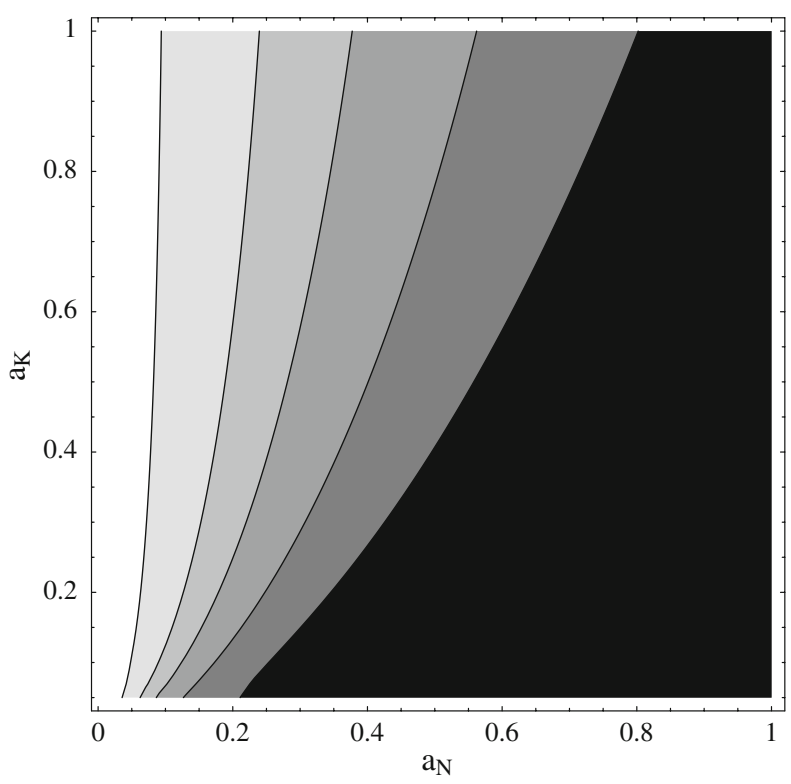

Fig. 1 The sets of sustainable actions for $\alpha=0.75,2.5,5,10,20,100 \%$. Increasing values of $\alpha$ correspond to a darker coloring
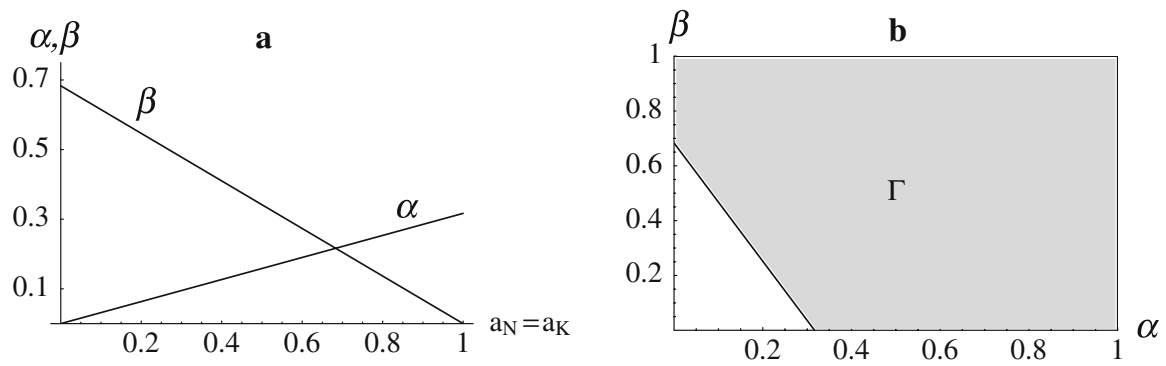

Fig. 2 a The probabilities of being ex post inefficient $(\beta)$ and of harming the future individual $(\alpha)$ for all possible "most efficient sustainable actions." b The set $\Gamma$ of feasible combinations of $(\alpha, \beta)$

Figure 2 depicts the risk of ex post inefficiency for all "most efficient sustainable actions," which are given by $a_{K}=a_{N}$ with $a_{N} \in[0,1]$, as well as the implied risk of harming the future individual. As it shows, there is a genuine trade-off between efficiency and sustainability. Also, the feasible combinations of sustainability and ex post efficiency have the properties asserted by Proposition 5: $\Gamma$ is a compact set with a decreasing lower boundary $\beta^{*}(\alpha)$, and $\beta^{*}(\alpha)$ is strictly decreasing in $\alpha$ as long as $\beta^{*}(\alpha)>\beta_{\min }$. The minimal achievable probability of being ex post inefficient is $\beta_{\text {min }}=0$ and it can be attained whenever the value of $\alpha$ is higher than $\approx 0.33$.

Finally, it can be easily shown that all "most efficient sustainable actions," that is, all $\left(a_{K}, a_{N}\right)$ with $a_{K}=a_{N}$, lie on the efficient frontier, as Proposition 7 suggests.

In addition to illustrating the results of the preceding sections, this example also serves to highlight the impact of uncertainty. The linear production and preference 
structure render the setup of this example favorable for the concept of weak sustainability, which holds that preserving a suitable aggregate of natural and man-made capital meets the requirement of sustainability. Under certainty, a linear aggregate $X=x_{K}+x_{N}$ would suffice to this end.

This does not hold under uncertainty. Maintaining a linear aggregate, that is, moving on a line $a_{K}=a_{N}$, assures only a very modest requirement of sustainability. The curves delineating the levels of sustainability as depicted in Fig. 1 show that at least a nonlinear aggregate is needed. Completely using up the natural capital stock (as allowed by a linear aggregation rule) is only sustainable at $\alpha \approx 33 \%$, as Fig. 2 shows. So under uncertainty, weak sustainability is indeed a rather weak obligation.

The other extreme-strong sustainability-would correspond to $a_{N}=0$. According to Fig. 2, this would imply a probability of about $70 \%$ of foregoing actions that benefit everyone and would thus be highly inefficient. So without explicitly considering uncertainty, we are unable to reach a reasonable balance between the objectives of sustainability and efficiency.

\section{Conclusions}

In this article, we have analyzed the question of how the long-run effects of present actions on future individuals can be evaluated if there is uncertainty and if intergenerational welfare comparisons are not feasible. For this, we have used the concept of sustainability advanced in Krysiak and Krysiak (2006), which avoids such comparisons, and explored its relation to a corresponding concept of ex post efficiency. We have provided a formal analysis of these concepts in a fairly general framework and have covered many important points not addressed in Krysiak and Krysiak (2006), like the structure of the sets of sustainable and efficient actions, the relation between sustainability and efficiency, and the possibility to reduce the conflict between these concepts by focusing on specific sustainable actions.

Our results show that it is not only possible but even advantageous to study questions of intertemporal distribution in a risk-based framework. Despite the complications arising from preference and outcome uncertainty, the basic elements of our analysis, like the set of sustainable actions, the set of efficient actions, and the definition of "most efficient sustainable actions," have convenient mathematical properties that facilitate their use in a welfare analysis or in a social choice setup. Thus in this framework, sustainability can easily be used as an a priori constraint in economic analysis, as discussed in Howarth (1995) and Woodward (2000). Furthermore, due to the uncertainty, we get a continuous dependency of the sets of allowable actions on the levels of sustainability and efficiency and a continuous trade-off between these requirements. Thus, the bivariate rankings ("sustainability" vs. "non-sustainability") of the deterministic case are changed to gradual characterizations. The incompatibility of sustainability and efficiency is also replaced by a smooth trade-off that leaves ample room for balancing these objectives.

Of course, these advantages come at some costs. We have used rather restrictive assumptions on the expectations concerning future preferences and outcomes to assure that the distribution problem remains analytically tractable. We doubt that 
these assumptions can be significantly relaxed without sacrificing important properties of our framework. But they are substantially more general than the model of an infinitely lived individual that is conventionally used in the sustainability discourse, which assumes certainly known and unchanging preferences.

Another objection to our framework might be that it requires a choice between sustainability and efficiency, that is, the level of sustainability $(\alpha)$ has to be set. Clearly, this is a decision with important ethical implications. But although it might be hard to settle on an ethically tenable level of $\alpha$, this gradual choice should be much simpler, at least from an ethical perspective, than the choice between sustainability without efficiency and efficiency without sustainability that is implicit in most deterministic frameworks.

\section{References}

Arrow, K.J., Fisher, A.C.: Environmental preservation, uncertainty, and irreversibility. Q J Econ 88, 312-319 (1974)

Asheim, G.B., Brekke, K.A.: Sustainability when capital management has stochastic consequences. Soc Choice Welf 19, 921-940 (2002)

Asheim, G.B., Buchholz, W., Tungodden, B.: Justifying sustainability. J Environ Econ Manag 41, 252-268 (2001)

Asheim, G.B., Tungodden, B.: Resolving distributional conflicts between generations. Econ Theory 24, 221-2301 (2004)

Ayong Le Kama, A.: Preservation and uncertain future preferences. Econ Theory 8, 745-752 (2001)

Ayong Le Kama, A., Schubert, K.: Growth, environment and uncertain future preferences. Environ Resour Econ 28, 31-53 (2004)

Baldursson, F.M., Magnusson, G.: Portfolio fishing. Scand J Econ 99, 389-403 (1997)

Brundtland, G: The U.N. Commission on Environment and Development: Our Common Future. Oxford: Oxford University Press (1987)

Dharmadhikari, S., Joag-Dev, K.: Unimodality, Convexity and Applications. New York: Academic Press (1988)

Edwards, S.F., Link, J.S., Rountree, B.P.: Portfolio management of wild fish stocks. Ecol Econ 49, 317-329 (2004)

Fisher, A.C., Krutilla, J.V.: Valuing long-run ecological consequences and irreversibilities. J Environ Econ Manag 1, 96-108 (1974)

Foley, D.: Resource allocation and the public sector. Yale Econom Essays 7, 45-98 (1967)

Heal, G.M., Chichilnisky, G., Beltratti, A.: Uncertain future preferences and conservation. In: Chichilnisky, G., Vercelli, A. (eds.) Sustainability: Dynamics and Uncertainty. Kluwer, Dordrecht (1998)

Howarth, R.B.: Sustainability under uncertainty: a deontological approach. Land Econ 71, 417-427 (1995)

Howe, R.E.: Sections and extensions of concave functions. J Math Econ 16, 53-64 (1987)

Krysiak, F.C., Krysiak, D.: Sustainability with uncertain future preferences. Environ Resour Econ 33, 511-531 (2006)

Markowitz, H.M., Todd, G.P.: Mean-Variance Analysis in Portfolio Choice and Capital Markets. New York: Wiley (1987)

Riley, J.G.: The just rate of depletion of a natural resource. J Environ Econ Manag 7, 291-307 (1980)

Samuelson, P.A.: The fundamental approximation theorem of portfolio analysis in terms of means, variances, and higher moments. Rev Econ Stud 37, 537-542 (1970)

Springer, U: International diversification of investments in climate change mitigation. Ecol Econ 46, 181193 (2003)

Woodward, R.T.: Sustainability as intergenerational fairness: efficiency, uncertainty, and numerical methods. Am J Agric Econ 82, 581-593 (2000) 